\title{
miR-93-5p attenuates apoptosis and autophagy by regulating SRY-box2 in spinal cord-injured rats in vivo and PC12 cells in vitro
}

Yan An, Jianing Li, MS Da He, Mingxing Fan

Department of Spine Surgery, Beijing Jishuitan Hospital, Beijing, China

Submitted: 29 March 2020

Accepted: 6 July 2020

Arch Med Sci

DOI: https://doi.org/10.5114/aoms.2020.98142

Copyright $\odot 2020$ Termedia \& Banach

\begin{abstract}
Introduction: In the current work we studied the involvement of miR-93-5p in spinal cord injury $(\mathrm{SCl})$ by involving $\mathrm{SCI}$ rats and LPS-mediated injuries in PC12 cells.

Material and methods: The PC12 cells were exposed to LPS in order to induce cell injury that mimicked the in vitro model of spinal cord injury. The in vivo model was created by submitting rats to contusion injury. The cell apoptosis and viability were evaluated by flow cytometry and cell counting kit (CCK-8). The expression of various apoptosis and autophagy-related proteins was done by western blot assay.

Results: The treatment of LPS caused cell injuries in PC12 cells. The levels of miR-93-5p were suppressed in LPS-injured cells. Over-expression of miR93-5p decreased autophagy and apoptosis and increased cell viability in LPS injured cells via up-regulation of SRY-box2. Up-regulation of SRY-box2 ameliorated LPS-mediated cell injury whereas suppression of SRY-box2 accelerated the LPS-mediated cell injury. Also, upregulation of miR-93-5 $p$ blocked the JAK/STAT and MAPK/ERK pathways via up-regulating the expression of SRY-box2. Treatment in SCl rats with miR-93-5p agomir inhibited cell apoptosis and autophagy.

Conclusions: The results of the study confirmed the attenuating role of miR93-5p against cell autophagy and apoptosis in LPS-injured PC12 cells and $\mathrm{SCl}$ rats via up-regulation of the expression of SRY-box 2 .
\end{abstract}

Key words: miR-93-5p, PC12 cells, spinal cord injury, SRY-box2.

\section{Introduction}

Spinal cord injury $(\mathrm{SCl})$ is an undesired condition with a high mortality rate, it also has a significant influence on human health, affecting life expectancy and quality [1]. There are reports about the existence of two different mechanisms contributing in the pathogenesis of $\mathrm{SCl}$ : the first mechanism involves primary mechanical injury, followed by secondary injury, which involves numerous interconnected damaging actions including biochemical imbalances, and altered vascular as well as cellular responses [2, 3]. The second phase of spinal cord injury is associated with a number of biological reactions, which include apoptosis, neuronal damage, and inflammation [4-6]. For the treatment of SCl it is crucial for clinicians to understand its progression and the involved molecular mechanism. MiRNAs (miRs) are small, endogenous, non-cod-

\author{
Corresponding author: \\ Da He MS \\ Department \\ of Spine Surgery \\ Beijing Jishuitan Hospital \\ Beijing 100035 \\ China \\ E-mail: 734034461@qq.com
}


ing RNAs measuring about 20-25 nucleotides in length, which are responsible for altering the regulation of genes at the post-transcriptional level by cleaving the messenger RNA [7]. Many recent studies have confirmed the potential role of miRs in a number of cellular processes, such as differentiation, proliferation, invasion, migration, and cell metastasis in various malignancies [8]. Other recent studies have confirmed that miRs are responsible for regulating the majority of human genes and could affect signalling pathways responsible for pathological responses post $\mathrm{SCl}[9,10]$.

MiR-93-5p is a member of the miR-106b 25 cluster and is found to be up-regulated in a number of cancer conditions [11-13]. miR-93-5p has been found to be involved in the modulation of the process of angiogenesis via inhibition of the expression of LATS2 in astrocytoma [14]. In addition to this, miR-93-5p has also been reported to regulate cellular processes such as promotion of proliferation in cancer [14]. Moreover, studies have recently demonstrated that miR-93-5p suppresses differentiation and migration of neurons [15]. However, the role of miR-93-5p in SCl and the involved signalling pathway remain unclear.

Looking into the involvement of miR-93-5p in various processes associated with neuronal cells, we aimed to screen its functioning in LPS-mediated injuries in $\mathrm{SCl}$ rats and $\mathrm{PC} 12$ cells. We also investigated the involved pathway. For this study we selected PC12 cells, also called pheochromocytoma cells, which show similar characteristics to those of neurons; these cells also had characteristics common to neuroendocrine cells. These cells also have the very important quality of being sub-cultured very easily compared with $\left.\right|^{\text {ry }}$ neurons $[16,17]$. We showed that the expression of miR-93-5p was suppressed in SCl rats, and in LPS-induced PC12 cells up-regulation of miR-93$5 p$ improved the LPS induced injuries in the PC12 cells. Furthermore, we found that SRY-box 2 was up-regulated by miR-93-5p; it was also shown that there was a cross-regulation between SRYbox2 and miR-93-5p in LPS-injured PC12 cells. It was also demonstrated that expression levels of both SRY-box2 and miR-93-5p were suppressed in $\mathrm{SCl}$ rats. In addition to this, miR-93-5p mimic caused up-regulation of SRY-box2 and inhibited the neuronal apoptosis as well as autophagy in $\mathrm{SCl}$ rats.

\section{Material and methods}

\section{Experimental groups and animal model of $\mathrm{SCl}$}

For the study we selected Sprague-Dawley (SD) male rats weighing between 190 and $220 \mathrm{~g}$. All the animals were housed in controlled tem- perature conditions and were kept in polypropylene cages with free access to water and palette diet. The animals were provided by the animal centre of Beijing Jishuitan Hospital China. The animal protocols were approved by the ethical clearance board of Beijing Jishuitan Hospital China; the approval number was BJ0114C. A total of 54 rats were used, among which 32 were divided into four groups comprising eight rats per group, named as the sham group $(n=8), \mathrm{SCl}$ for 1 day $(n=8), \mathrm{SCl}$ for 3 days $(n=8)$, and $\mathrm{SCl}$ for 7 days $(n=8)$. The remaining 24 rats were used for the study of over-expression of miR-93-5p on levels of SRY-box 2, autophagy, and apoptosis-related proteins post spinal cord injury. The rats were divided into three groups as the saline (vehicle) group $(n=8)$, the agomir-negative control group (NC) $(n=8)$, and the miR-93-5p agomir $(n=8)$ group.

To induce $\mathrm{SCl}$, the rats were anaesthetised by phenobarbital injection, then the backs of rats were shaved to remove hairs and fixed in a prone position. A small incision was made in the skin at the T9-T10 position along with the spinous process using ophthalmic scissors; a contusion injury was induced by putting the rats in a weight drop apparatus with a weight of 8 grams at a height of $40 \mathrm{~mm}$. The confirmation of $\mathrm{SCl}$ was done by observing specific symptoms such as spinal cord haemorrhage, oedema, tail showing spastic swing, flaccid paralysis of hind limbs, and flutter [18].

\section{Intrathecal injection of miR-93-5p agomir}

For intrathecal injection of miR in $\mathrm{SCl}$ rats, laminectomy (partial) was done at T12/T13 and an intrathecal catheter was introduced. The miR-93-5p agomir and negative controls were procured from RiboBio, China. The agomir NC and agomir were given by intrathecal route: briefly, the miR NC and agomir were solubilised in phosphate-buffered saline ( $0.9 \%$ sodium chloride) and injected continuously at a rate $1 \mu \mathrm{l} / \mathrm{h}$ with the help of osmotic mini pumps implanted subcutaneously into the intrathecal space post SCI for three days [19]. The process was carried out by delivery pumps, which were primed for $12 \mathrm{~h}$ at $37^{\circ} \mathrm{C}$ to insure immediate delivery post implantation. The muscles were sutured, and then the skin was also sutured followed by intraperitoneal instillation of $5 \mathrm{ml}$ lactated Ringer's solution. After performing the surgeries, the rats were given penicillin $G$ for three days to prevent infection.

\section{Harvesting of $\mathrm{SCl}$ samples}

The experimental rats were sacrificed on day 1 , day 3 , and day 7 post $\mathrm{SCl}$ by high-dose pentobarbital injection $(200 \mathrm{mg} / \mathrm{kg})$. The spinal cord tissues 
were harvested from the operated region from all the groups. The spinal cord tissues were flash frozen using liquid nitrogen at $-80^{\circ} \mathrm{C}$ followed by qRT-PCR analysis or western blot assay.

\section{Cell culture and treatment of LPS}

For the in vitro study we selected the PC12 cells (ATCC, USA), which were maintained in DMEM media (ThermoFisher USA) supplemented by foetal bovine serum (10\%), $100 \mathrm{U} / \mathrm{ml}$ penicillin, and $100 \mu \mathrm{g} / \mathrm{ml}$ streptomycin under humid conditions with $5 \% \mathrm{CO}_{2}$ and a temperature of $37^{\circ} \mathrm{C}$. The cells were allowed to reach $80 \%$ of confluence and were starved using DMEM media along with FBS $(0.1 \%)$ for 60 min followed by treatment with LPS in predefined serial concentration for $12 \mathrm{~h}$.

\section{Transfection of miRs}

The miR-93-5p inhibitor, mimic, or corresponding negative control constructs (NC) were procured from RiboBio China. The PC12 cells were subjected to seeding in six-well plates prior to transfection and were then transfected by miR-93-5p inhibitor, mimic, and NC upon reaching confluence of $50 \%$. The process of cell transfection was done using Lipofectamine-3000 reagent (Invitrogen, USA) as per the supplied instructions. The cells were processed $48 \mathrm{~h}$ post transfection. The sequences of mimic, inhibitor, or corresponding NC are depicted in Table I. The evaluation was performed by a microplate reader (Biotek, USA). To study the consequences of SRY-box 2 and miR-93-5p on the cell viability, the $P C 12$ cells received transfection of miR-93-5p inhibitor, mimic, or corresponding negative control or pcPSEN1, shPSEN1, or respective controls. Furthermore, the transfected cells were treated with LPS $(5 \mu \mathrm{g} / \mathrm{ml})$ for $12 \mathrm{~h}$ followed by measurement of the cell viability.

\section{Quantitative real-time PCR studies}

The RNA was isolated from the target tissues and transfected cells with the help of TRIzol reagent following the supplied instructions. The isolated RNA was submitted for reverse transcription onto cDNA using reverse transcription kit. The levels of SRY-box2 and miR-93-5p were analyzed by qRTPCR SYBR geen mixture and Taqman mRNA assay respectively following the provided instructions. GAPDH and U6 were selected as loading control for mRNA and miRNA respectively. The sequences of primers used for the study are presented in Table II.

\section{Cell viability assay}

Viability of cells was analysed by CCK- 8 assay (SigmaAldrich, USA) as per the supplied instructions. Briefly, after transfection, PC-12 cells were incubated in 96-well plates with $1 \times 10^{4}$ cells in each well at $37^{\circ} \mathrm{C}$ in an incubator. The wells were added with the supplied reagent $(10 \mu \mathrm{l})$, and the plate was incubated again for $1 \mathrm{~h}$ at $37^{\circ} \mathrm{C}$. The optical density was measured of each well at $450 \mathrm{~nm}$ using a micro-plate reader (Bio-Tek Inst., USA). The percentage cell viability was calculated as the mean optical density of transfected cells/ mean optical density of the control group $\times 100 \%$.

\section{Cell apoptosis study}

The cell apoptosis was evaluated with the help of an Annexin-V-FITC and propidium iodide (PI) apoptosis kit (ThermoFisher, USA) following the supplied instructions. The PC12 cells were harvested and rinsed at least three times with phosphate-buffered saline solution (PBS). The cells were again re-suspended using binding buffer $(500 \mu \mathrm{l})$ and were added with Annexin-V-FITC and $\mathrm{PI}(5 \mu \mathrm{l})$ for $30 \mathrm{~min}$ at room temperature and in dark conditions. The extent of cell apoptosis was evaluated by flow cytometry (BD Bioscience, USA).

Table I. Sequences of miR used in the study

\begin{tabular}{|lc|}
\hline Name & Primer sequences \\
\hline miR-93-5p mimic & $\begin{array}{l}\text { Sense: GATGGACGTGCTTGTCGTGAAAC } \\
\text { Antisense: GGACTTGGCGTTGCCAAACA }\end{array}$ \\
\hline miR-93-5p inhibitor & UCAAGCUGGACAGGUCAGGGC \\
\hline miR-93-5p mimic control & Sense: GATGGAGCTGCGTTCCGTGACAA \\
& Antisense: GGACTTCGGGTTGCCAACAC \\
\hline miR-93-5p inhibitor control & UCAGCUGAGGGCAACACGGCG \\
\hline
\end{tabular}

Table II. The primer sequence SRY-box2/miR-93-5p for PCR analysis

\begin{tabular}{|lcc|}
\hline \multirow{2}{*}{ Genes } & \multicolumn{2}{c|}{ Primer sequence } \\
\cline { 2 - 3 } & Forward & Reverse \\
\hline miR-93-5p & AAGUGCUGUUCGGCAGUAGUG & UUCACGACGAGUCGUCAUCAC \\
\hline SRY-box2 & CGCGGATCCATGTACAACATGATGGAGACGGAGC & CCGGTTCGATTTATCGCGTCGACTCACATG \\
\hline
\end{tabular}




\section{Western blot analysis}

Both the spinal cord tissue and PC12 cells were treated with RIPA buffer along with protease inhibitors. The proteins were estimated using BCA protein assay kit (ThermoFisher, USA). The proteins $(30 \mu \mathrm{g})$ were loaded on an NuPAGE Bis-Tris gel system (ThermoFisher, USA) and then transferred to polyvinylidene difluoride (PVDF) membranes. The membranes were blocked with bovine serum albumin (5\%) (Cell Siganling Tech, USA) for $1 \mathrm{~h}$ and washed three times with TTBS buffer. The membranes were treated and incubated with respective primary antibodies: anti-Bax (1:1000), anti-Bcl-2 (1: 1000), anti-GAPDH (1: 1000), anti-caspase-3/-9 (1: 1000), anti-Beclin-1 (1: 1000), anti-LC (1: 1000), anti-SRY-box 2 (1: 1000), antip-ERK (1 : 1000), anti-p62 (1 : 1000), anti-pMAPK (1 : 1000), anti-ERK (1 : 1000), anti-MAPK (1 : 1000), anti-p-STAT1 (1 : 1000), anti-STAT1 (1 : 1000), anti-JAK1 (1: 1000), anti-p-JAK1 $(1: 1000)$, anti-STAT3 (1: 1000), and antip-STAT3 (1: 1000). All the antibodies were bought from Cell Signaling

A

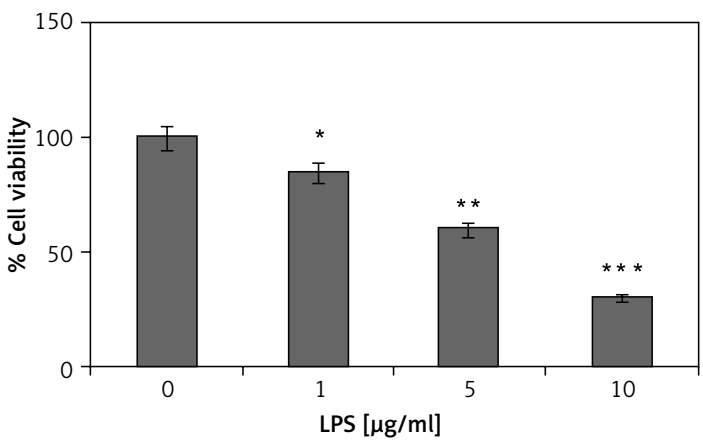

C

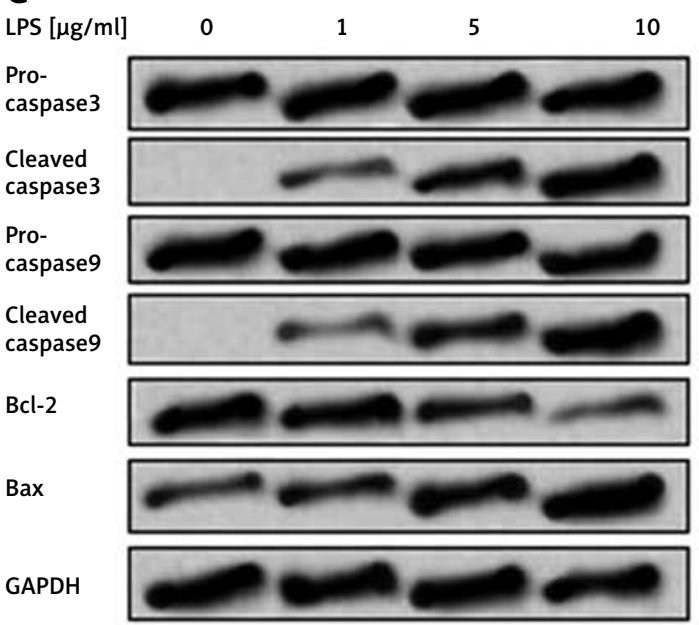

Tech, USA. The membranes were rinsed at least three times with TTBS and were then again incubated with horseradish peroxidase secondary antibody (Sigma-Aldrich, USA) with dilution of $1: 5000$ for $2 \mathrm{~h}$ at room temperature. The developed blots were viewed using Image Lab software.

\section{Statistical analysis}

The results are presented as mean \pm SD; all the statistical anyses were performed by GraphPad Prism software (GraphPad USA). The $p$-values were obtained by one-way analysis of variance (ANOVA); the values $<0.05$ were regarded as statistically significant.

\section{Results}

Treatment of LPS caused autophagy and apoptosis in PC12 cells

It was observed that increasing the dose of LPS in PC12 resulted in a significant decrease in viability of PC12 cells (Figure $1 \mathrm{~A}$ ). Treatment of

B

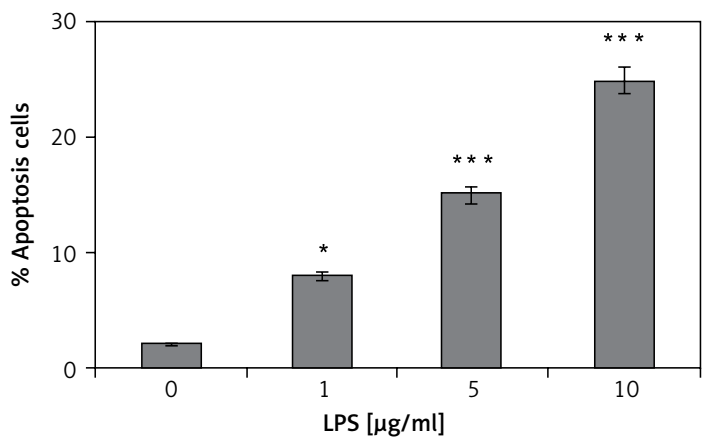

\section{D}

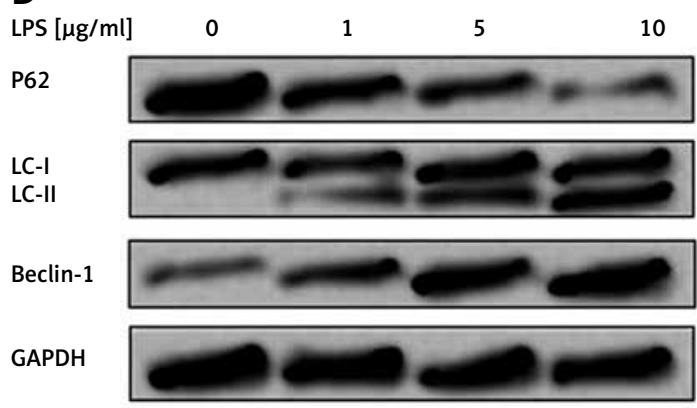

Figure 1. Treatment of LPS resulted in cell autophagy and apoptosis in PC12 cells. The PC12 cells were exposed to series of concentrations of LPS of 1,5 , and $10 \mu \mathrm{g} / \mathrm{ml}$ for $12 \mathrm{~h}$. A - Cell Counting Kit- 8 assay was done for evaluating viability of cells. B - Rate of cell apoptosis was analysed by flow cytometry for Annexin V-FITC/PI staining. C - Western blot analysis was done for expression of apoptosis-related protein. D - Expression of autophagy-related proteins by western blot analysis

${ }^{\star} P<0.05,{ }^{* *} p<0.01,{ }^{* * *} p<0.001$. 
LPS resulted in apoptosis of PC12 cells with increasing dose, which also resulted in an increased apoptosis rate in cells (Figure $1 \mathrm{~B}$ ). The treatment of LPS also resulted in increased protein levels of crucial pro-apoptosis factors such as cleaved caspase-9/-3 and Bax and decreased the levels of $\mathrm{BCl}-2$ (Figure $1 \mathrm{C}$ ). Moreover, exposure of LPS caused suppression of $\mathrm{p} 62$ and over-expression of Beclin-1 and LC-II (Figure 1 D). Because LPS at the concentration of $5 \mu \mathrm{g} / \mathrm{ml}$ resulted in a substantial decrease in viability and rate of apoptosis in cells, we selected this concentration for our further experiments. The outcomes of this experiment confirmed that LPS caused cell injury in PC12 cells.

\section{MiR-93-5p attenuated LPS-mediated} injuries in PC12 cells

To study the outcomes of miR-93-5p on LPS-mediated injuries in PC12 cells, we trans-

A

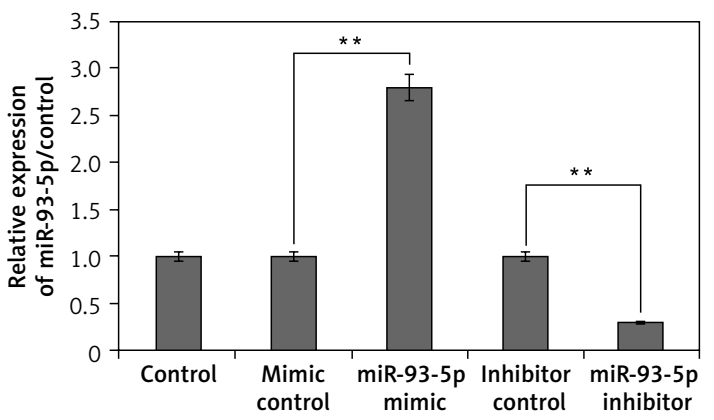

$c$

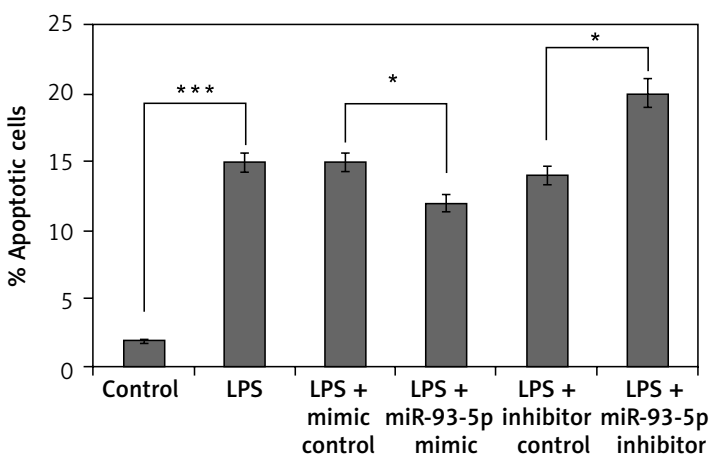

E

Beclin-1

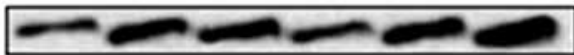

LC-I

LC-II

p62

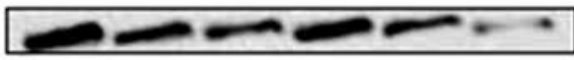

GAPDH

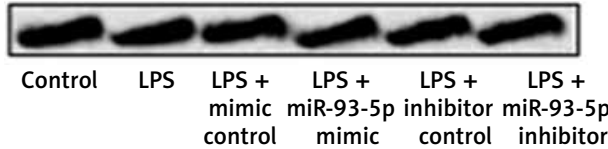

fected the cells with miR-93-5p mimic or inhibitor; the transfection was confirmed by GRT-PCR analysis. As postulated, the expression of miR93-5p increased significantly post transfection with mimic and was suppressed upon transfection of inhibitor (Figure $2 \mathrm{~A}$ ). As observed, miR93-5p mimic caused a significant increase in cell viability in LPS-exposed PC12 cells, whereas the transfection of miR-93-5p inhibitor decreased the cell viability (Figure 2 B). Furthermore, we evaluated the functional significance of miR93-5p on autophagy and apoptosis in LPS-injury PC12 cells. We observed that transfection of miR-93-5p mimic caused a significant decrease in the rate of apoptosis in cells, whereas the miR93-5p inhibitor enhanced the rate of apoptosis (Figure 2 C). Similarly, miR-93-5p mimic caused over-expression of specific anti-apoptotic proteins (Bcl-2, pro-caspase-3/-9) and suppressed

B

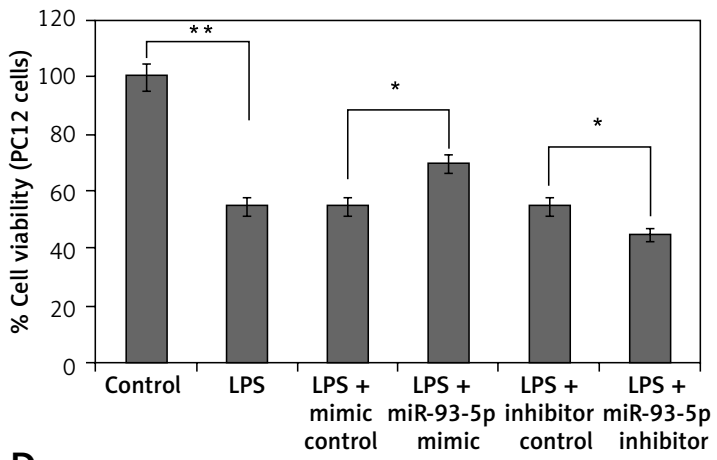

D

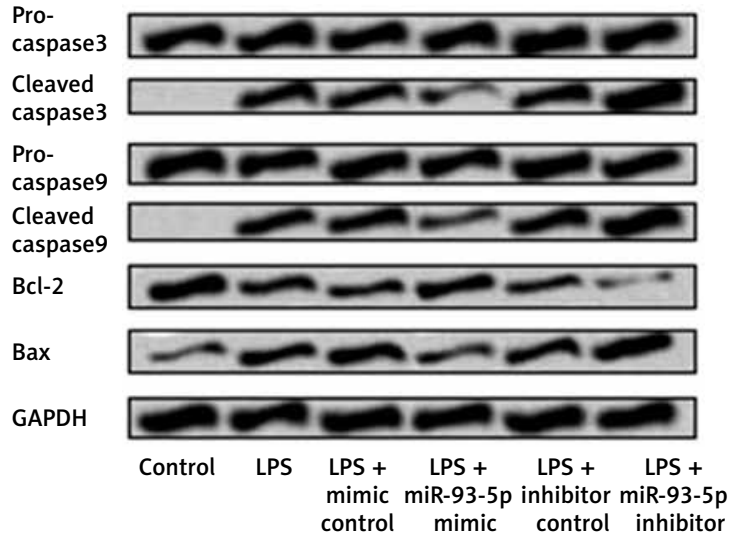

Figure 2. MiR-93-5p modulated LPS-mediated cell injury in PC12 cells. The PC12 cells received transfection of miR-93-5b inhibitor, mimic, and their respective controls. The cells were incubated with LPS $(5 \mu \mathrm{g} / \mathrm{ml})$ for $12 \mathrm{~h}$. A - qRT-PCR analysis was done to verify the efficiency of transfection. B - Results of cell viability. C - Results of rate of cell apoptosis (late). D - Expression levels of apoptosis-related proteins evaluated by western blot analysis, cell counting kit-8, and flow cytometry. E - The outcomes of western blot analysis for expression of proteins of autophagy-related proteins

${ }^{*} p<0.05,{ }^{* *} p<0.01,{ }^{* * *} p<0.001$. 
the expression of pro-apoptotic factor (cleaved caspase-3/-9, Bax) (Figure 2 D). It was also observed that the over-expression of miR-93-5p after transfection of mimic inhibited the levels of Beclin-1 and LC-II and increased the levels of p62 in LPS-induced cells. However, miR-93-5p inhibitor demonstrated the opposite effects on these factors. These findings indicate that miR-93-5p exerts an attenuating effect on LPS-mediated cell injury in PC12 cells.

\section{miR-93-5p caused upregulation of SRY-box 2 in PC12 cells}

To confirm whether miR-93-5p regulated the levels of SRY-box2 in PC12 cells, they were transfected with miR-93-5p mimics, inhibitor, and respective controls. It was observed that cells receiving transfection of miR-93-5p inhibitor showed suppressed protein as well as mRNA levels of SRY-box 2 (Figure 3). However, the opposite effect was observed in cells that received transfection of miR-93-5p mimic, suggesting that SRY-box 2 was positively correlated with miR-93-5p.

miR-93-5p inhibitor induced LPS-mediated cell injures in PC12 cells via suppression of SRY-box 2

To investigate whether miR-93-5p modulated the autophagy and apoptosis in PC12 cells via SRYbox 2 pathway, the PC12cells were transfected with vector and shRNA selective SRY-box 2. As observed, the protein as well as mRNA expression levels of SRY-box 2 were significantly increased after the cells were transfected with pEX-SRY-box 2, whereas the levels decreased after transfection with shSRY-box 2 (Figure 4 A). We showed that miR-93-5p inhibitor reversed the effects on autophagy and apoptosis rate by up-regulating the expression of SRY-box 2 when transfected with pEX-SRY-box2 (Figures $4 \mathrm{~B}$ and $\mathrm{C}$ ). In addition to this, we observed that over-expression of SRY-box 2 decreased the levels

A

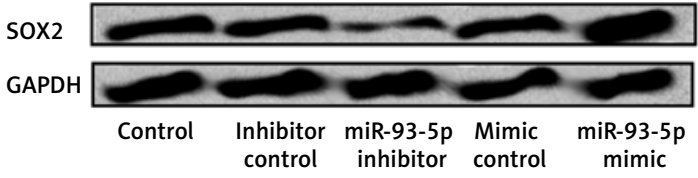

Figure 3. MiR-93-5p resulted in over-expression of SRY-box2 in PC12 cells. The cells received transfection of miR 93-5p inhibitor, mimic, and respective controls. The mRNA as well as protein expression of SRY-box2 were evaluated by $q R T-P C R$ and western blot analysis

${ }^{\star} P<0.05,{ }^{* *} p<0.01$. of autophagy-related factors Beclin-1 and LC-II, and increased the expression of p62 (Figure 4 D). These outcomes indicated that inhibition of miR93-5p may induce LPS-mediated cell injury in PC12 cells via suppression of SRY-box 2.

\section{SRY-box 2 participated in LPS-mediated cell injury in PC12 cells}

To study the role of SRY-box 2 in LPS-mediated injuries in PC12 cells, the cells were transfected with pEX-SRY-box 2 and sh-SRY-box 2 followed by an autophagy and viability study. The outcomes suggest that inhibition of SRY-box-2 increases LPS-mediated cell injury and decreases cell viability, apoptosis, and autophagy (Figures 5 A-D). However, the opposite effect was observed in cells transfected with pEX-SRY-box 2 (Figures 5-D). These findings indicate that SRY-box 2 is involved in regulation of LPS-mediated injury in cells.

miR-93-5p blocked the MAPK/ERK and JAK/ STAT cascade via upregulation of SRY-box2

In the current study we in investigated the effect of miR-93-5p on JAK/STAT and MAPK/ERK cascade in LPS-injured cells. We showed that miR-93-5p mimic suppressed the levels of $p$-ERK as well as P-MAPK, and it was observed that the effect was reversed upon inhibition of SRY-box2. It was also observed that over-expression of miR93-5p decreased the levels of p-JAK1, p-STAT1, and p-STAT3 (Figure $6 \mathrm{~A}$ ). However, the regulating action of miR-93-5p on JAK/STAT signalling cascade was halted by inhibition of SRY-box2 (Figure $6 \mathrm{~B}$ ). These findings suggest that up-regulation of miR-93-5p blocked JAK/STAT and MAPK/ERK cascade via up-regulation of SRY-box2 cascade.

miR-93-5p agomir inhibited cell autophagy and apoptosis in spinal cord-injury rats

To validate the in vitro findings, we investigated the effect of miR-93-5p and SRY-box2 on spinal

B

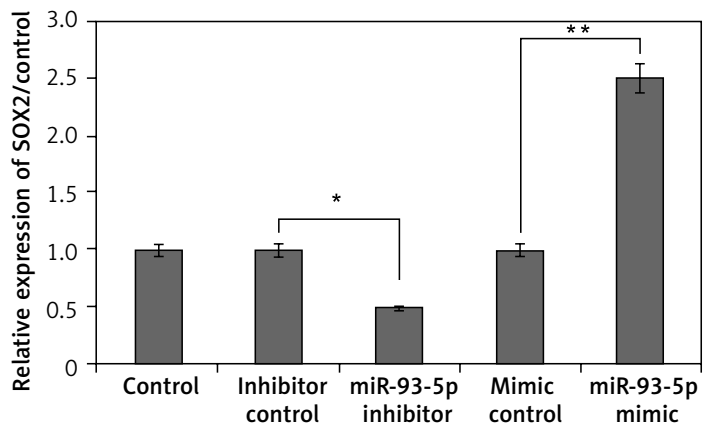


A

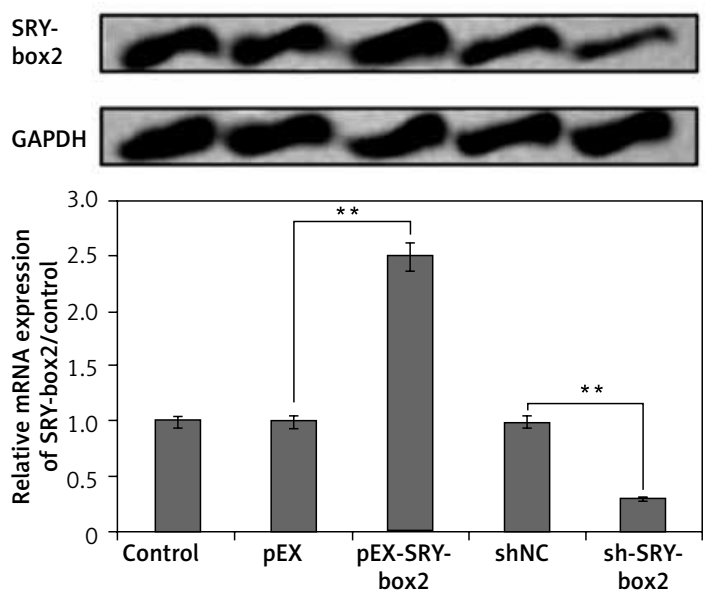

C

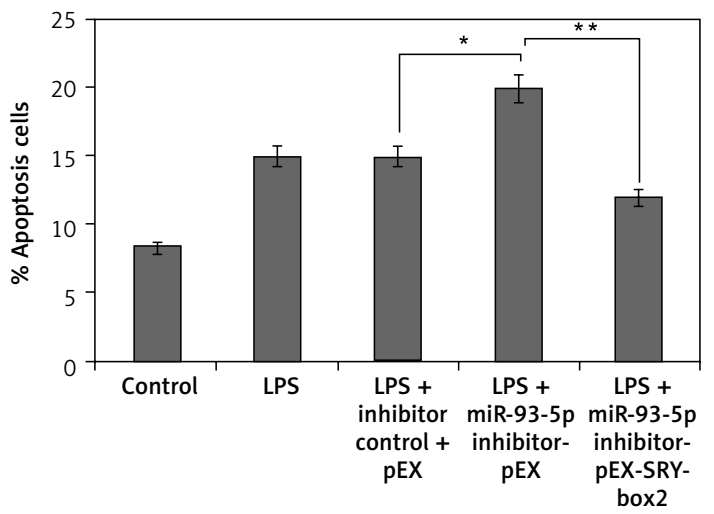

B

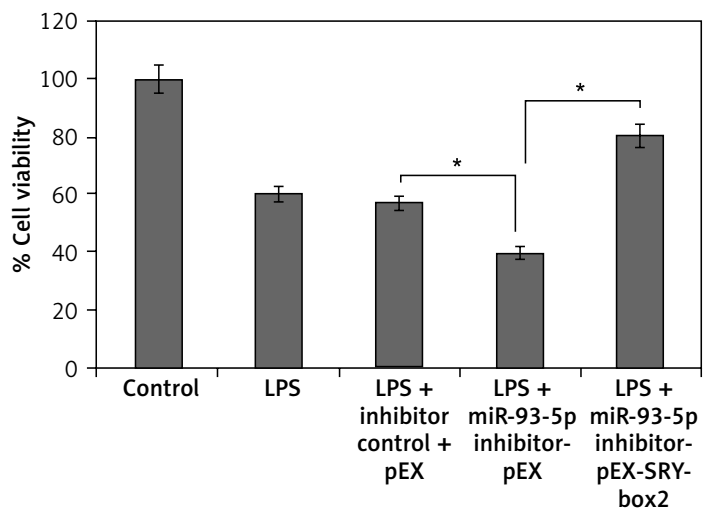

D

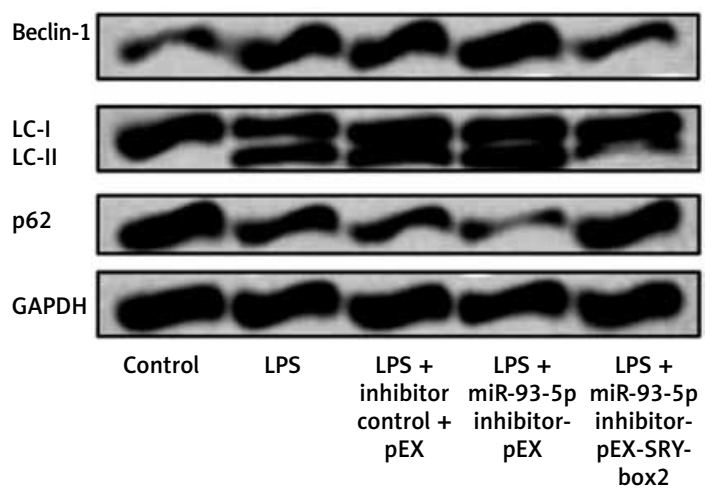

Figure 4. MiR-93-5p inhibitor induced LPS-mediated injures in PC12 cells via suppression of SRY-box2. The cells received transfection of $\mathrm{pEX}-\mathrm{SRY}$-box2, sh-SRY-box2, and their respective controls or co-transfection with miR-93$5 p$ inhibitor and pEX-SRY-box2; after this the cells were exposed to LPS $(5 \mu \mathrm{g} / \mathrm{ml})$ for $12 \mathrm{~h}$. A - qRT-PCR and western blot analysis was done to assess the efficiency of transfection. Results of cell viability (B) and the rate of apoptosis cell rate (C) was assessed by CCK-8. D - Western blot analysis for expression of autophagy-related proteins

${ }^{\star} P<0.05,{ }^{* *} p<0.01$.

cord-injury rats. The expression of miR-93-5p and SRY-box2 in $\mathrm{SCl}$ rats was studied by qRT-PCR. It was observed that the spinal cord tissues of $\mathrm{SCl}$ rats showed significantly suppressed levels of miR-93-5p on the first, third, and seventh day post spinal cord injury (Figure $7 \mathrm{~A}$ ). In addition to this, SRY-box2 levels were significantly decreased in spinal cord injury rats on the first, third, and seventh day post spinal cord injury (Figure 7 B). The $\mathrm{SCl}$ rats were injected with miR-93-5p agomir for inducing up-regulation of miR-93-5p, which was screened by qRT-PCR. The outcomes of qRTPCR suggested that levels of miR-93-5p were significantly over-expressed in rats when injected with miR-93-5p agomir (Figure 7 C). In addition to this in our study, we evaluated miR-93-5p agomir on levels of SRY-box2 in $\mathrm{SCl}$ rats. The outcomes indicated that injecting miR-93-5p agomir resulted in up-regulation of both mRNA and protein levels of SRY-box2 (Figures 7 D, E). Furthermore, the study also evaluated the efficacy of miR-93$5 p$ agomir on autophagy and apoptosis in spinal cord tissue cells. It was observed that, miR-93-5p agomir caused a significant decrease in the rate of apoptosis and attenuated the expression of apoptosis-related proteins (Figures 7 F, G). MiR-93-5p agomir also suppressed the levels of Beclin- 1 and LC-II and increased the levels of p62 (Figure $7 \mathrm{H}$ ). The findings of in vivo experiments suggest that up-regulated levels of miR-93-5p also decrease autophagy and apoptosis in cells as well as in $\mathrm{SCl}$ rats.

\section{Discussion}

Increasing studies have suggested abnormal expression of miRs in spinal cord injury, which leads to the alteration of the expression of genes and affects the associated signalling pathways [20, 21]. Studies also have revealed that miRs play important roles in various pathological procedures and responses of spinal cord injury $[22,23]$. Therefore, miRs are believed to be potential therapeutic targets involved in functional recovery after spinal 
A

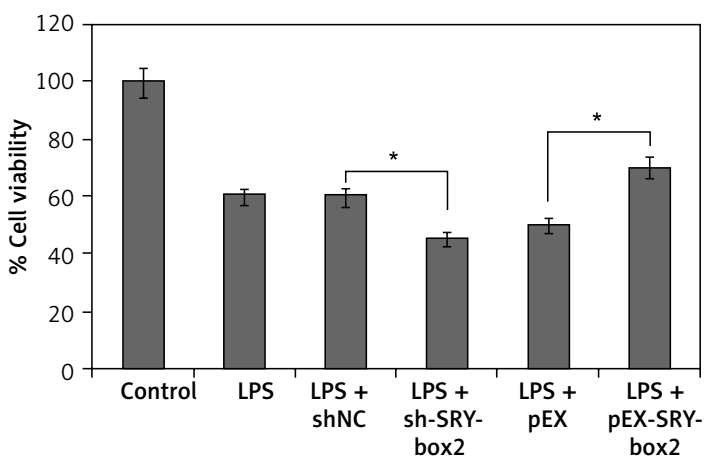

C

Control LPS LPS + LPS + LPS + LPS +

ShNC sh-SRY- pEX pEX-SRY

Pro-

caspase3

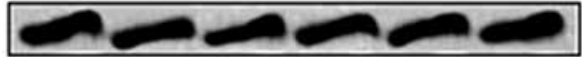

Cleaved caspase 3

Pro-

caspase9

Cleaved caspase 9
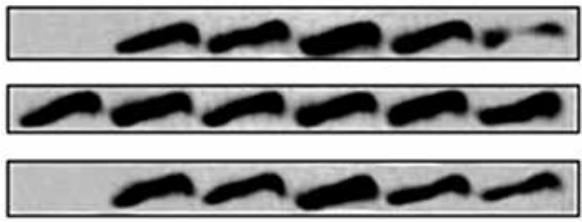

$\mathrm{Bcl}-2$

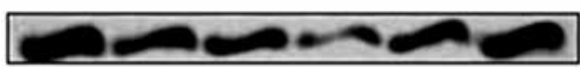

Bax

GAPDH
B

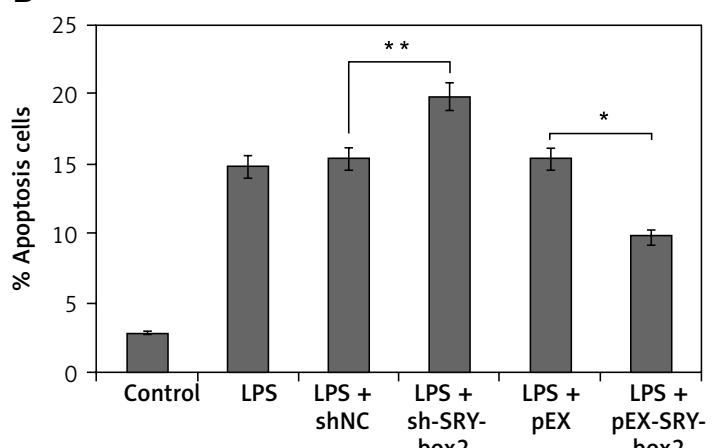

D

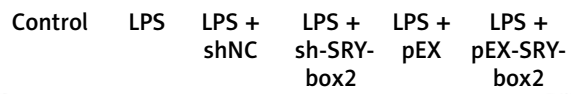

Beclin-1

p62

LC-I

LC-II

GAPDH

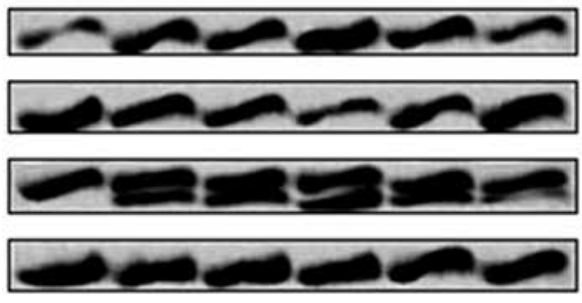

Figure 5. SRY-box2 was associated in LPS-mediated cell injuries in PC12 cells. The PC12 cells received transfection with pEX-SRY-box2, sh-SRY-box2, or respective controls; after this the cells were exposed to LPS (5 $\mu \mathrm{g} / \mathrm{ml})$ for $12 \mathrm{~h}$. A - Results of cell viability. B - Rate of apoptosis cell rate. C - The expressions of apoptosis-related proteins were evaluated by CCK-8 assay, Western blot, and flow cytometry. D - The expressions of autophagy-related protein were evaluated by Western blot analysis

${ }^{\star} P<0.05,{ }^{* *} p<0.01$.

A

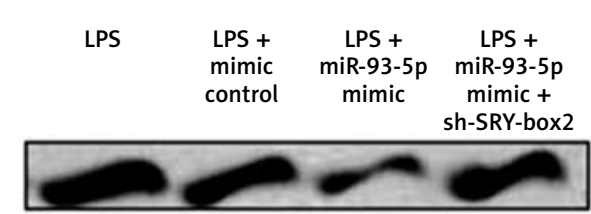

MAPK

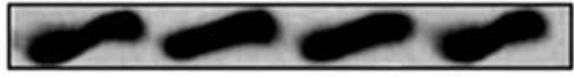

p-ERK

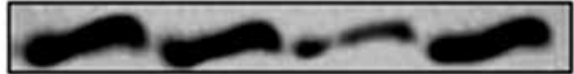

ERK

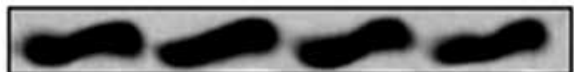

GAPDH

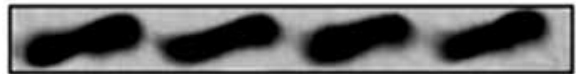

B

P-STAT

p-STAT1

STAT1

p-STAT3

STAT3

p-JAK1

JAK1
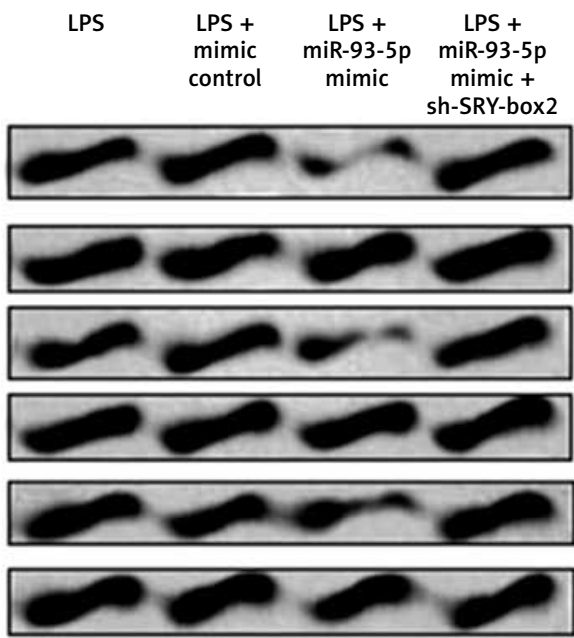

GAPDH

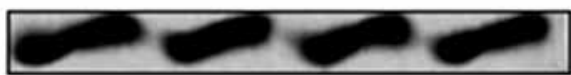

Figure 6. MiR-93-5p blocked JAK/STAT pathway and MAPK/ERK pathway via up-regulation of SRY-box2. PC12 cells were transfected with miR-103 mimic and mimic control, or co-transfected with sh-SOX2. Cells were incubated with $5 \mathrm{\mu g} / \mathrm{ml}$ LPS for $12 \mathrm{~h}$. The protein expressions of core factors related with MAPK/ERK signalling pathway (A) and JAK/STAT signalling pathways (B) were measured by western blot 
A

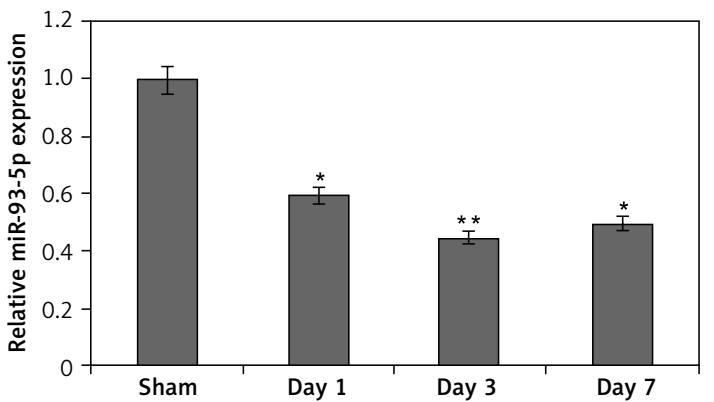

C

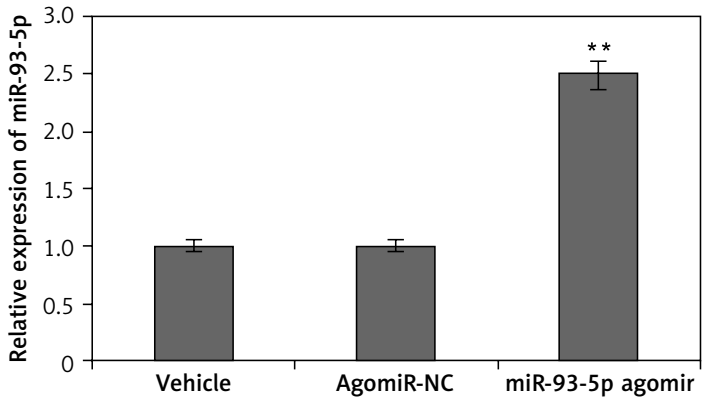

E
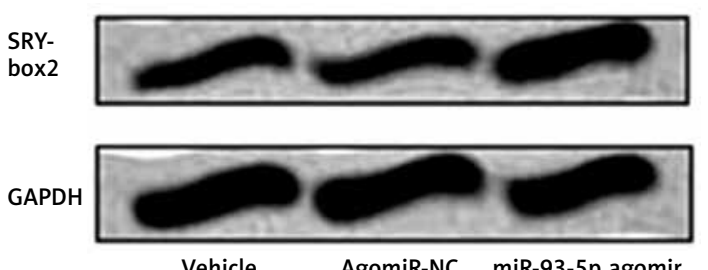

G
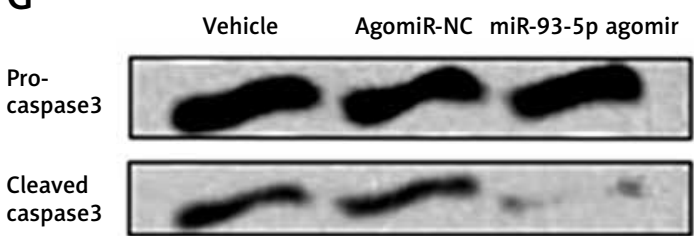

Pro-

caspase9

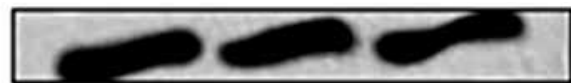

Cleaved

caspase9

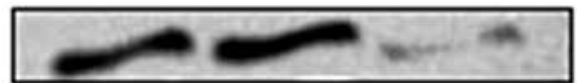

Bcl-2

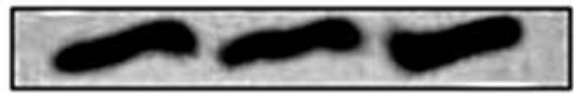

Bax

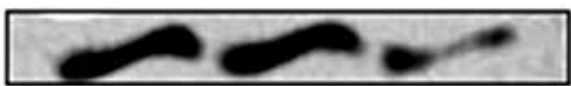

GAPDH

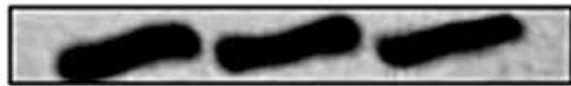

\section{B}

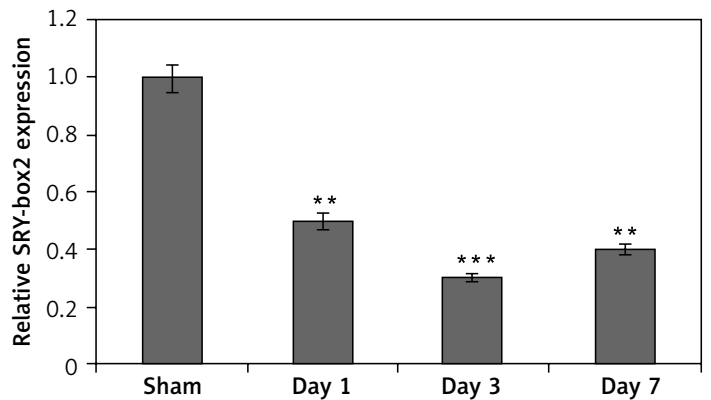

D

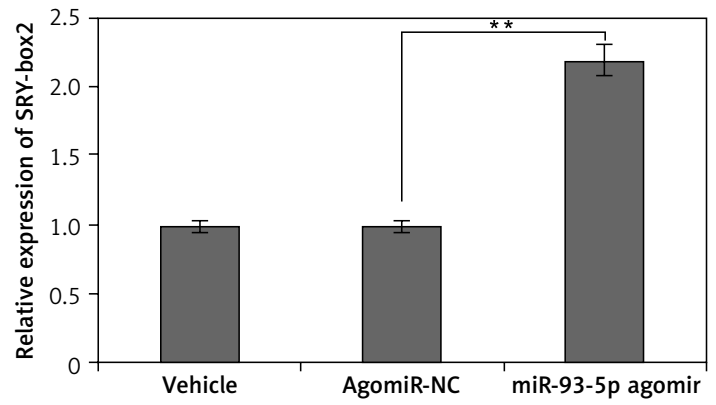

F

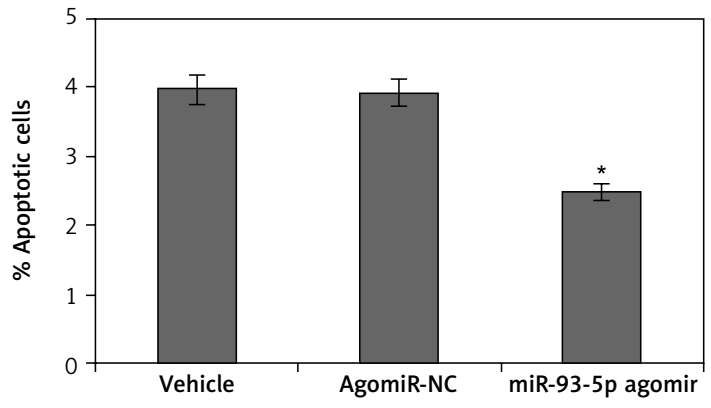

G

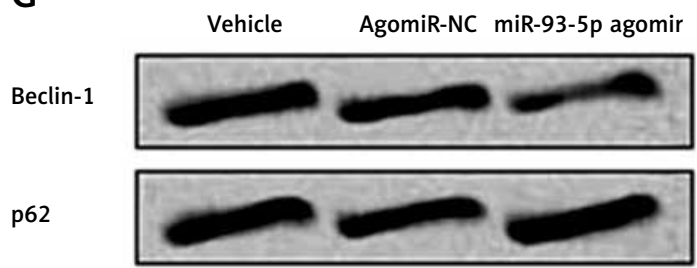

LC-I

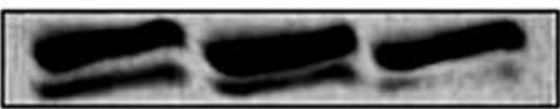

GAPDH

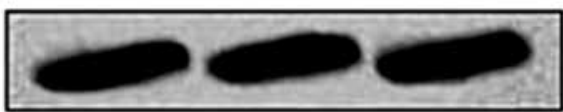

Figure 7. MiR-93-5p agomir halted cell autophagy and apoptosis in spinal cord-injury rats. A - The spinal cord-injury rats were assessed by western blot analysis for expression of miR-93-5p after day 1 , day 3, and day 7 . B - The spinal cord tissues of SCl rats were assessed by western blot analysis for expression of SRY-box 2 after day 1, day 3 , and day 7. C - Effect of miR-93-5p agomir on levels of miR-93-5p by qRT-PCR in SCl rats ( $7^{\text {th }}$ day). The mRNA (D) and and protein levels of SRY-box2 (E) in SCI rats post treatment with miR-93-5p agomir and assessed by qRT-PCR and western blot. $\mathrm{F}$ - Quantitative results of showing percentage apoptosis of cells from rats submitted to $\mathrm{SCI}$ after treatment of miR-93-5p agomir injection. The levels of apoptosis-related $(\mathbf{G})$ and autophagy-related proteins evaluated by western blot assay (H) 
cord injury. In the current study, we showed that up-regulation of miR-93-5p caused a significant increase in cell viability, and decreased autophagy and apoptosis in PC12 cells subjected to LPS-mediated cell injury, whereas suppression of miR-93-5p increased the rate of LPS-mediated cell injury in PC12 cells. In addition to this, we found that miR93-5p attenuated the cell injury via up-regulation of the expression of SRY-box 2; also, in vivo studies in spinal cord injury rats suggest that the levels of both miR-93-5p and SRY-box2 are suppressed in $\mathrm{SCl}$ rats. Also, miR-93-5p agomir caused up-regulation of SRY-box2 and inhibited cell autophagy as well as apoptosis in spinal cord-injured rats. These results suggested that mir-93-5p can be an important therapeutic target in LPS-mediated cell injury in $\mathrm{PC} 12$ cells and in $\mathrm{SCl}$ rats.

According to reports, miR-93-5p has been associated with the development and progression of multiple cancers such as breast cancer, colon cancer, ovarian cancer, and lung cancer [24]. Recently, a study suggested that miR-93-5p suppressed the differentiation and migration of neurons [15]. In addition, another study suggested that miR-93-5p decreased the VEGF and IL-8-dependent angiogenesis in neuroblastoma cells [25]. miR-93-5p has also been shown to affect autophagy in retinal ganglionic cells via AKT/mTOR pathway [26]. Our findings suggest that miR-93-5p decreases the apoptosis cell rate and suppresses the expression of pro-apoptotic factors and autophagy-related proteins in PC12 submitted to LPS-mediated injury. Also, the expression levels of miR-93-5p were suppressed in rats submitted to spinal cord injury by contusion whereas miR-93-5p agomir decreased the cell autophagy and apoptosis in $\mathrm{SCl}$ rats. These results confirm the involvement of miR-93-5p in $\mathrm{SCl}$ and its role in decreasing apoptosis.

SRY-box 2 is a protein belonging to the SOX family of genes, and it has been found to be necessary for the survival of the early mouse embryo and is reported to possess an MHG-box DNA-binding domain that regulates Fgf-4 gene [27]. SRY-box2 is crucial in the process of neuronal cell differentiation and is responsible for the process of self-renewal of stem cells $[28,29]$. In a study, deletion of neuron-specific SRY-box2 resulted in impairment of brain development in embryos [30]. Also, SRY-box2 has been shown to be an oncogene in numerous cancer conditions, which could lead to migration and proliferation of cells [31]. The outcomes of the present study suggest that SRY-box2 is associated with LPS-mediated cell injury in PC12 cells. We also showed that miR-93-5p resulted in up-regulation of SRY-box2, which caused attenuation of cell viability, and decreased autophagy and apoptosis of neuron-like PC12 cells. Taken together, miR-93-5p may reduce LPS-mediated injury in PC12 cells via up-regulation of SRY-box 2 .
Janus kinase (JAK), one of the members of the family of non-receptor tyrosine kinases, acts as an activator and transducer of STAT cascade, and it is one of the most crucial mediators for transducing a signal from the surface of the cell to the nucleus [32]. Activation of JAK/STAT cascade is reported to be involved in differentiation of neurons or glial cells in the development of CNS [33]. Earlier Studies, have demonstrated that IL- 6 is responsible for activation of JAK/STAT pathway in neurons of spinal cord-injured rats [34]. Activation of MAPK pathway has been reported to be involved in $\mathrm{SCl}$ [35]. Also, it was found that up-regulation of SRYbox2 inhibits the expression of p38MAPK cascade in osteoblast cells [36]. In addition to this, MAPK has been found to activate a number of proteins such as kinase in the STAT/JAK cascade [37]. Looking into this, we postulated that miR-93-5p could be involved in regulation of MAPK/ERK and JAK/ STAT cascade involving SRY-box2. We showed that over-expression of miR-93-5p inhibited the levels of p-STAT1, pSTAT3, and p-JAK, which was blocked by suppression of SRY-box2. Similarly, it was observed that miR-93-5p over-expression suppressed MAPK/ERK cascade mediated by suppression of SRY-box2. These findings clearly indicate that miR-93-5p may lead to suppression of JAK/ STAT and MAPK/ERK cascade via up-regulation of SRY-box2, and this may be responsible for the attenuating effect of miR-93-5p in spinal cord injury.

In conclusion, the findings of the present work suggest that miR-93-5p corrected LPS-mediated cell autophagy and apoptosis through over-expression of SRY-box 2 in PC12 cells. The possible cascade associated with the attenuating effect of miR-93-5p on autophagy and apoptosis may be JAK/STAT and MAPK/ERK, as confirmed in both in vitro and in vivo models. The outcomes of the present work might be helpful in understanding the mechanisms involved in $\mathrm{SCl}$ and might also act cumulatively in developing potential therapeutic approaches for treating SCl. Also, we concluded that miR-93-5p may play an important role in treating and designing therapy for $\mathrm{SCl}$. Furthermore, more detailed studies are required to explore the role of miR-93-5p and SRY-box2 on the JAK/STAT and MAPK/ERK pathways in spinal cord injury models.

\section{Conflict of interest}

The authors declare no conflict of interest.

\section{References}

1. Lee BB, Cripps RA, Fitzharris M, Wing PC. The global map for traumatic spinal cord injury epidemiology: update 2011, global incidence rate. Spinal Cord 2014; 52: 110-6.

2. Yunta M, Nieto-Díaz M, Esteban FJ, Caballero-López M, Navarro-Ruíz R, Reigada D. MicroRNA dysregulation in 
the spinal cord following traumatic injury. PLoS One 2012; 7: e34534.

3. Nieto-Diaz M, Esteban FJ, Reigada D, Muñoz-Galdeano T, Yunta M, Caballero-López M. MicroRNA dysregulation in spinal cord injury: causes, consequences and therapeutics. Front Cell Neurosci 2014; 8: 53.

4. Ning B, Gao L, Liu RH, Liu Y, Zhang NS, Chen ZY. microRNAs in spinal cord injury: potential roles and therapeutic implications. Int J Biol Sci 2014; 10: 997-1006.

5. Bareyre FM, Schwab ME. Inflammation, degeneration and regeneration in the injured spinal cord: insights from DNA microarrays. Trends Neurosci 2003; 26: 555-63.

6. Nesic O, Svrakic NM, Xu GY, et al. DNA microarray analysis of the contused spinal cord: Effect of NMDA receptor inhibition. J Neurosci Res 2002; 68: 406-23.

7. Krichevsky AM. MicroRNA profiling: from dark matter to white matter, or identifying new players in neurobiology. Sci World J 2007; 7: 155-66.

8. Li Z, Yu X, Shen J, Law PTY, Chan MTV, Wu WKK. MicroRNA expression and its implications for diagnosis and therapy of gallbladder cancer. Oncotarget 2015; 6: 13914-21.

9. Friedman RC, Farh KK, Burge CB, Bartel DP. Most mammalian mRNAs are conserved targets of microRNAs. Genome Res 2008; 19: 92-105.

10. Hu JZ, Huang JH, Zeng L, Wang G, Cao M, Lu HB. Antiapoptotic effect of microRNA-21 after contusion spinal cord injury in rats. J Neurotrauma 2013; 30: 1349-60.

11. Deng Z, Qian J, Liu FQ, et al. Expression level of miR-93 in formalin-fixed paraffin-embedded tissues of breast cancer patients. Genet Test Mol Biomark 2014; 18 366-70.

12. Kawano M, Tanaka K, Itonaga I, Ikeda S, Iwasaki T, Tsumura H. microRNA-93 promotes cell proliferation via targeting of PTEN in osteosarcoma cells. J Exp Clin Cancer Res 2015; 34: 76

13. Liang $\mathrm{H}$, Wang $\mathrm{F}$, Chu $\mathrm{D}$, et al. miR-93 functions as an oncomiR for the downregulation of PDCD4 in gastric carcinoma. Sci Rep 2016; 6: 23772.

14. Fang L, Du WW, Yang W, et al. MiR-93 enhances angiogenesis and metastasis by targeting LATS2. Cell Cycle 2012; 11: 4352-65.

15. Lattanzi A, Gentner B, Corno D, et al. Dynamic activity of miR-125b and miR-93 during murine neural stem cell differentiation in vitro and in the subventricular zone neurogenic niche. PLoS One 2013; 8: e67411.

16. Zhang H, Wu F, Kong X, et al. Nerve growth factor improves functional recovery by inhibiting endoplasmic reticulum stress-induced neuronal apoptosis in rats with spinal cord injury. J Transl Med 2014; 12: 130.

17. Li R, Yin F, Guo YY, Zhao KC, Ruan Q, Qi YM. Knockdown of ANRIL aggravates $\mathrm{H} 2 \mathrm{O} 2$-induced injury in $\mathrm{PC}-12$ cells by targeting microRNA-125a. Biomed Pharmacother 2017; 92: 952.

18. Zhou Y, Cui Z, Xia X, et al. Matrix metalloproteinase-1 (MMP-1) expression in rat spinal cord injury model. Cell Mol Neurobiol 2014; 34: 1151-63.

19. Liebscher T, Schnell L, Schnell D, et al. Nogo A antibody improves regeneration and locomotion of spinal cord-injured rats. Ann Neurol 2005; 58: 706-19.

20. Hutchison ER, Okun E, Mattson MP. The therapeutic potential of microRNAs in nervous system damage, degeneration, and repair. Neuromolecular Med 2009; 11: 153-61.

21. Nakanishi K, Nakasa T, Tanaka N, et al. Responses of microRNAs 124a and 223 following spinal cord injury in mice. Spinal Cord 2010; 48: 192-6.
22. An Y, Li J, Yuan Q, Fan M. MicroRNA-466c-3p exerts protective effect on neuronal apoptosis and improves functional recovery post spinal cord injury via mitochondrial apoptotic pathway. AMB Expr 2010; 10: 113.

23. Hao J, Jin X, Shi Y. Zhang H. miR-93-5p enhance lacrimal gland adenoid cystic carcinoma cell tumorigenesis by targeting BRMS1L. Cancer Cell Int 2018; 18: 72.

24. Fabbri E, Montagner $\mathrm{G}$, Bianchi N, et al. MicroRNA miR93-5p regulates expression of IL-8 and VEGF in neuroblastoma SK-N-AS cells. Oncol Rep 2016; 35: 2866-72.

25. Li R, Jin Y, Li Q, Sun X, Zhu H, Cui H. MiR-93-5p targeting PTEN regulates the NMDA-induced autophagy of retinal ganglion cells via AKT/mTOR pathway in glaucoma. Biomed Pharmacother 2018; 100: 1-7.

26. Gubbay J, Collignon J, Koopman P, et al. A gene mapping to the sex-determining region of the mouse $Y$ chromosome is a member of a novel family of embryonically expressed genes. Nature 1990; 346: 245-50.

27. Pevny LH, Nicolis SK. Sox2 roles in neural stem cells. Int J Biochem Cell Biol 2010; 42: 421-4.

28. Episkopou V. SOX2 functions in adult neural stem cells. Trends Neurosci 2005; 28: 219-21.

29. Miyagi S, Masui S, Niwa $\mathrm{H}$, et al. Consequence of the loss of Sox 2 in the developing brain of the mouse. FEBS Lett 2008; 582: 2811-5.

30. Jiang J, Li Z, Yu C, Chen M, Tian S, Sun C. MiR-1181 inhibits stem cell-like phenotypes and suppresses SOX2 and STAT3 in human pancreatic cancer. Cancer Lett 2015; 356: 962-70.

31. Kim NH, Lee MY, Park SJ, Choi JS, Oh MK, Kim IS. Auranofin blocks interleukin-6 signalling by inhibiting phosphorylation of JAK1 and STAT3. Immunology 2007; 122: 607-14.

32. Tsuda M, Kohro Y, Yano T, et al. JAK-STAT3 pathway regulates spinal astrocyte proliferation and neuropathic pain maintenance in rats. Brain 2011; 134: 1127-39.

33. Yamauchi K, Osuka K, Takayasu M, et al. Activation of JAK/STAT signalling in neurons following spinal cord injury in mice. J Neurochem 2006; 96: 1060-70.

34. Xu Z, Wang BR, Wang X, et al. ERK1/2 and p38 mitogen-activated protein kinase mediate iNOS-induced spinal neuron degeneration after acute traumatic spinal cord injury. Life Sci 2006; 79: 1895-905.

35. Ding D, Xu H, Liang Q, Xu L, Zhao Y, Wang Y. Over-expression of Sox 2 in $\mathrm{C} 3 \mathrm{H} 10 \mathrm{~T} 1 / 2$ cells inhibits osteoblast differentiation through Wnt and MAPK signalling pathways. Int Orthop 2012; 36: 1087-94.

36. Collins CS, Hong J, Sapinoso L, et al. A small interfering RNA screen for modulators of tumor cell motility identifies MAP4K4 as a promigratory kinase. Proc Natl Acad Sci USA 2006; 103: 3775-80. 